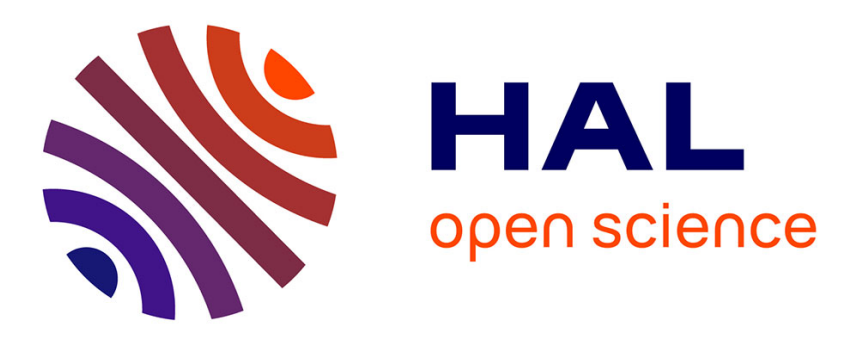

\title{
Scaled-up Helical Nanobelt Modeling and Simulation at Low Reynolds Numbers.
}

\author{
Tiantian Xu, Gilgueng Hwang, Nicolas Andreff, Stéphane Régnier
}

\section{To cite this version:}

Tiantian Xu, Gilgueng Hwang, Nicolas Andreff, Stéphane Régnier. Scaled-up Helical Nanobelt Modeling and Simulation at Low Reynolds Numbers.. IEEE International Conference on Robotics and Automation, ICRA'12., May 2012, RiverCentre, Saint Paul, Minnesota, United States. pp.4045-4051. hal-00768520

\section{HAL Id: hal-00768520 \\ https://hal.science/hal-00768520}

Submitted on 21 Dec 2012

HAL is a multi-disciplinary open access archive for the deposit and dissemination of scientific research documents, whether they are published or not. The documents may come from teaching and research institutions in France or abroad, or from public or private research centers.
L'archive ouverte pluridisciplinaire HAL, est destinée au dépôt et à la diffusion de documents scientifiques de niveau recherche, publiés ou non, émanant des établissements d'enseignement et de recherche français ou étrangers, des laboratoires publics ou privés. 


\title{
Scaled-up Helical Nanobelt Modeling and Simulation at Low Reynolds Numbers
}

\author{
Tiantian Xu, Gilgueng Hwang, Nicolas Andreff and Stéphane Régnier
}

\begin{abstract}
Micro and nanorobots can change many aspects of medicine by enabling targeted diagnosis and therapy, and minimal invasive surgery. A helical nanobelt with a magnetic head was proposed as a microrobot driven by rotating magnetic field in prior works. Magnetically coated tails were already shown in some works. However the control of such surface magnetic tails is not clearly realized yet. This paper aims to obtain control parameters for the modeling and simulation of the influence of surface magnets onto the swimming performances. For this, we created scaled-up helical nanobelts and the experimental testbed to get the control parameters and to prepare future closed-loop control.
\end{abstract}

\section{INTRODUCTION}

Micro and nanorobots have the potential to change many aspects of medicine. Microrobots can be used for the localized delivery of chemical and biological substances (e.g. targeted drug delivery, hyperthermia), to remove material by mechanical means, to act as simple controllable static structures or to transmit biological data from a specific location hard to reach [1].

Swimming at the microscale is considered as swimming at low Reynolds numbers. Helical propeller inspired by nature's bacterial swimming behaviors is promising for medical microrobots, because helical propulsion is suited to a low Reynolds number regime [2], [3]. In Japan, researchers proposed the first spiral type swimming mechanism with a cubic magnetic head driven by a rotating magnetic field [4]. This micro swimming mechanism was as large as a few millimetres. This group tested the swimming performances of spiral type micro-machines at low Reynolds number. They predicted that this spiral type micro-machine with a length of $11.5 \mathrm{~mm}$ could still be scaled down to micrometer-sized machine [5].

Nanorobots are significantly smaller than microrobots, and typically envisioned as devices to target individual cells [6]. Researchers are not yet ready to fabricate a nanorobot capable of performing even a

Manuscript received September, 2011. This work was supported by Émergence-UPMC-2011

T. XU is with Institut des Systèmes Intelligents et Robotique, UPMC, Paris, France. tiantian.xulisir.upmc.fr

G. Hwang is with Laboratoire de Photonique et de Nanostructures, Marcoussis, France. gilgueng.hwang@lpn.cnrs. fr

N. Andreff is with FEMTO-ST Institute, ENSMM, Besancon, France. nicolas. andreffefemto-st. fr

$\mathrm{S}$. Régnier is with Institut des Systèmes Intelligents et Robotique, UPMC, Paris, France. stephane.regnierdisir.upmc.fr simple medical task. They are still developing and characterizing the fundamental building blocks of future nanorobotic devices [1].

The first helical nanorobot called the "Artificial Bacterial Flagella" (ABF), with a length of approximately $50 \mu \mathrm{m}$ and a thickness of approximately $30 \mathrm{~nm}$ was developed by a group at ETH Zurich [7], [8], [9]. The ABF consists of a helical tail made by GaAs/InGaAs and a thin-square-plate soft-magnetic "head" on one end. A low-strength rotating magnetic field acts on $\mathrm{ABF}^{\prime} \mathrm{s}$ magnetic "head" to drive it. Further size reduction and applying the same principle to drive artificial magnetic propellers with a length of approximately $1 \mu \mathrm{m}$ was achieved in [10].

A group at ISIR and LPN demonstrated that electroosmotic propulsion of "Helical NanoBelt" (HNB) showed much higher swimming performances in terms of maximum swimming velocity and manipulation force. Moreover, a $10 \mathrm{~nm}$ thick ferromagnetic metal layer was successfully added to the surface of helical tails [11], [12]. This surface-coated HNBs has a similar geometry to $\mathrm{ABF}$, but their entire surface can be functional to propulsion in comparison to the ABF's soft magnetic head as the only functional part and the tail as a passive part.

Although all surface magnetic tails are advantageous, their control has not been clearly realized mainly due to the limited observation tools to characterize the swimming characteristics and to identify the control parameters in micro and nanoscale. This prevents their microrobotic applications which usually need to implement the closed loop control. In this paper, we propose to assemble Scaled-up Helical Nanobelts (SHNs) and to develop their control testbed to observe the propulsion characteristics (e.g. cut-off frequency, maximal translational speed) and to identify the control parameters (e.g. propulsion matrix) of helical microrobots. This proposed methods and system enable low-cost, simple geometry modifications, fast and easy control parameter identifications. This system reveals different propulsion characteristics and control parameters of two different SHNs with one or four pitch magnetic surfaces.

Section II presents an overview of the system including a control setup and SHNs used in the experiments. Section III introduces the control principle of the SHNs. Section IV presents the characteristics of the SHNs, including cut-off frequency, identification of 
the propulsion matrix, forces and torques analyses and translation tests.

\section{SYSTEM OVERVIEW}

This section presents an overview of our system. The latter (see Fig 1a) consists of a control system and a tube containing the SHN immersed in a viscous liquid named hereafter the target system. The control part controls the SHNs in the target part.

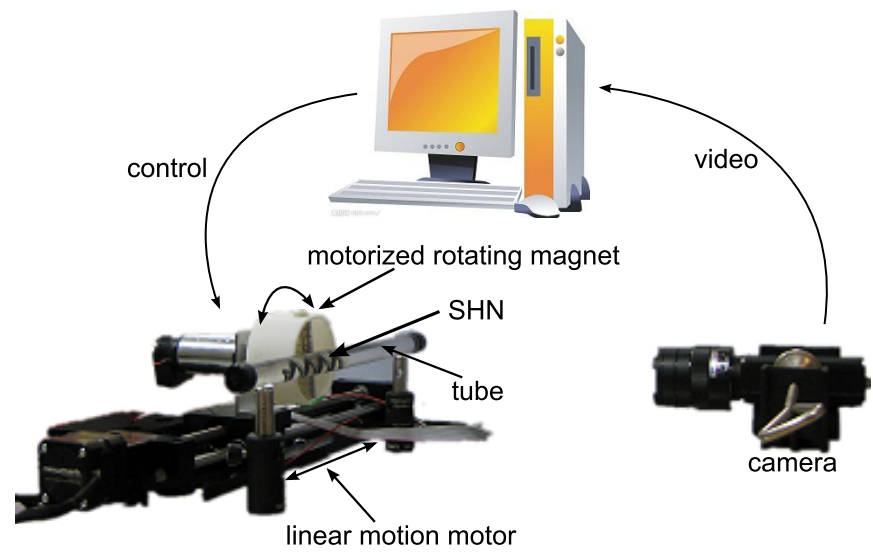

(a)

Fig. 1: (a) A global view of system. (b) Target system : scaled-up helical nanobelt encapsulated in a transparent tube filled with liquid.

\section{A. Control system}

Researchers utilized orthogonal arrangements of electromagnetic coil pairs to generate rotating uniform magnetic fields at the center of the system's workspace. However, these coil pairs are difficult to scale up [3]. A rotating permanent magnet allows us to control a scaled-up model wirelessly [13]. A linear motor stage contributes to give a translation motion.

The magnet used in the experiments is cylindrical, $60 \mathrm{~mm}$ in length and $15 \mathrm{~mm}$ in diameter, mounted on a Maxon DC Motor. The magnetic field magnitudes around the magnet were measured using a Hirst GM08 gaussmeter, and are depicted by Fig 2, in which the magnet is put along the $\mathrm{x}$ axis, and the center of the magnet is at the origin.

The rotating magnet manipulator is mounted on a Newmark System linear motion stage, whose travel range is $250 \mathrm{~mm}$ and maximal speed is $220 \mathrm{~mm} / \mathrm{s}$.

\section{B. Target system}

The target system is a SHN encapsulated in a transparent tube filled with viscous liquid. Liquids used in the experiment are different glycerol solutions.

The target system is placed from $1.5 \mathrm{~mm}$ to $3.5 \mathrm{~mm}$ away from the magnet axis. The magnetic field magnitude ranges from $10 \mathrm{mT}$ to $70 \mathrm{mT}$.

Two types of SHNs were fabricated for the experiments. The first type of SHNs named SHN1 (see

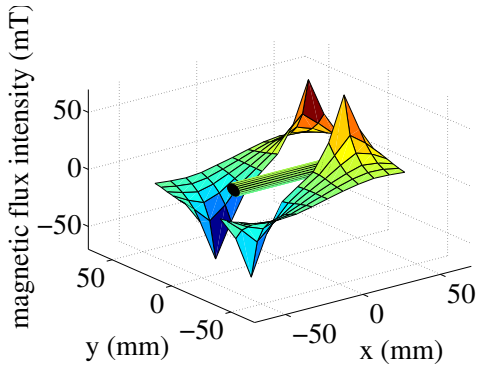

Fig. 2: Magnetic field magnitudes measured around the magnet. The black cylinder presents the magnet.

\begin{tabular}{|l|c|c|}
\hline Parameters & SHN1 & SHN4 \\
\hline Diameter & $6 \mathrm{~mm}$ \\
\hline Thickness & \multicolumn{2}{|c|}{$2 \mathrm{~mm}$} \\
\hline Pitch & \multicolumn{2}{|c|}{$20 \mathrm{~mm}$} \\
\hline Width & \multicolumn{2}{|c|}{$7.2 \mathrm{~mm}$} \\
\hline Nb of turns & \multicolumn{2}{|c|}{4.5} \\
\hline Length & \multicolumn{2}{|c|}{$97.2 \mathrm{~mm}$} \\
\hline Weight & $0.02 \mathrm{~N}$ & $0.05 \mathrm{~N}$ \\
\hline Volume & $1 \mathrm{~mL}$ & $1.5 \mathrm{~mL}$ \\
\hline Magnetic material & \multicolumn{2}{|c|}{$\mathrm{Ni}-\mathrm{Cu}-\mathrm{Ni}$} \\
\hline Magnetic layer & \multicolumn{2}{|c|}{$1 \mathrm{~mm}$} \\
\hline
\end{tabular}

TABLE I: Specifications of SHNs used in experiments.

Fig 3a) has its first pitch (i.e. a 1-full-turn portion of the helix) covered by small magnets (Q-05-1.5-01-N Supermagnete). The other one named SHN4 (see Fig $3 b)$ has its entire surface (i.e. the 4 pitches) covered by small magnets. The base structures of the SHNs nanobelt are made by a prototyping machine. The dimensions of SHNs are referred to the dimension of the real HNB [11]. The thickness of SHNs are limited by prototyping machine's capacity. The volumes of SHNs are measured by Archimede method. The dimensions and other specifications of the two types of SHNs used in the experiments are depicted by Table I.

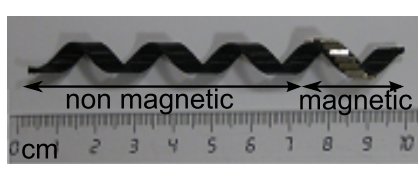

(a)

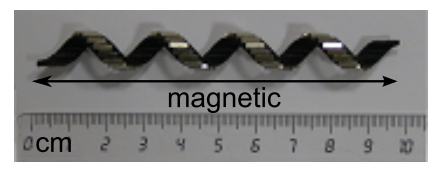

(b)
Fig. 3: (a) The surface of SHN1's first pitch is covered by magnets. (b) SHN4's entire surface is covered by magnets.

Swimming microscale objects are largely limited in their performances due to the low Reynolds dynamics [3]. The so-called Reynolds number, a dimensionless quantity that embodies the interaction between the inertia and viscosity of a fluid, is defined as :

$$
R e=\frac{\rho v L}{\mu}
$$

where $v$ is the velocity, $\rho$ is the density of the fluid, 


\begin{tabular}{|l|c|c|c|c|c|c|}
\hline type & HNB & \multicolumn{5}{|c|}{ SHNs } \\
\hline \% of glycerol & 0 & 0 & 50 & 75 & 90 & 95 \\
\hline Viscosity $(\mathrm{cP})$ & 1.308 & 1.308 & 9.01 & 65.2 & 498 & 1270 \\
\hline Reynolds number & 0.001 & 7 & 1 & 0.1 & 0.02 & 0.007 \\
\hline
\end{tabular}

TABLE II: Viscosities and Reynolds numbers for different glycerol solutions.

$L$ is a characteristic linear dimension (length of helical robot) and $\mu$ is the dynamic viscosity of the fluid.

In the following experiments, the liquids in which the SHNs swim are different glycerol solutions in order to analyse the influences of Reynolds numbers on the propulsion of SHNs. Table II summarizes the viscosities of different glycerol solutions at $10{ }^{\circ} \mathrm{C}$ and the Reynolds numbers for HNB in water and for SHNs in different glycerol solutions.

\section{Measurement system}

A Basler camera running at $60 \mathrm{~Hz}$ is used to measure the dropping velocities. The dropping velocities are calculated from the video recorded by the pixels travelled in unit time. The angular speeds are measured by the time that a SHN uses to finish a whole turn rotation.

\section{MODELLING AND CONTROL}

We demonstrate in this section the rotation principle by SHN1. Its magnetic pitch should be put in the area where the magnetic field is locally strong, called functional area. There are two functional areas in front of the magnet : on its left-hand side and on its right hand side, which are presented by Fig. 4a.

The magnet manipulator rotates in clockwise direction. The magnetic pitch is put in front of the magnet manipulator in the left-hand side, shown in Fig. 4e. Fig. 4a shows the magnetic field in the $y \mathrm{O} z$ plane generated by the magnet at positions 0 degree. All of the magnetic field vectors are in the $y O z$ plane. While the magnet rotates 30 degree, the magnetic field vectors lean down, as can be seen in Fig. $4 \mathrm{~b}$ and $4 \mathrm{c}$, and they tend to align an easy axis of the magnetic helical tail along the field. Although there are two possible easy axes (see Fig. 4d), the one that is closer to the local applied field acts as the easy axis, which is the first way of magnetization in Fig. $4 \mathrm{~d}$. This magnetization axis follows the local applied field to lean down. Therefore, the helical tail rotates in the counter clockwise direction of $z$ direction, seen by its head.

If the magnetic pitch is put in the right-hand side of the magnet manipulator, as presented by Fig. 4f, the second magnetization axis in Fig. $4 \mathrm{~d}$ acts as an easy axis, because it is closer to the local applied field described by Fig. 4c. In this case, the helical tail rotates in the clockwise direction, seen by its head. Finally, if the magnetic pitch is put in the middle area of magnet manipulator, the helical tail cannot rotate. These phenomena are proved by experiments.
For SHN4, it's enough that one of its pitches is in the functional area. The principle and the direction of rotation are the same as that of SHN1. But if two pitches of SHN4 are in the different functional areas of magnet, the SHN4 will be exerted two torques in opposite direction. Therefore, the SHN4 cannot rotate.

\section{A. Forces and torques}

Swimming along the axis of a helical propeller is known. The nonfluidic applied force $(f)$ and nonfluidic applied torque $(\tau)$ on a helical swimmer are linearly related to its velocity $(v)$ and angular speed $(\omega)$ with four principal quantities described by a symmetric propulsion matrix [2], [3] :

$$
\left[\begin{array}{l}
f \\
\tau
\end{array}\right]=\left[\begin{array}{ll}
a & b \\
b & c
\end{array}\right]\left[\begin{array}{l}
v \\
\omega
\end{array}\right]
$$

The parameters $a, b$, and c encapsulate the geometric and environmental properties of the helical robot. The nonfluidic applied forces for SHNs are gravitational, buoyancy, friction and magnetic forces while the only nonfluidic torque is the magnetic torque.

1) Apparent weight: The apparent weight is the net force between gravitational force and buoyancy force, which is defined as :

$$
\overrightarrow{f_{w}}=\left(\rho_{(S H N)}-\rho_{f}\right) \vec{g} V_{(S H N)}
$$

where $\rho_{(S H N)}$ is the density of the SHN, $\rho_{f}$ is the density of the fluid and $V_{(S H N)}$ is the volume of the SHN, which is shown in Table I.

2) Friction force: Friction is the force resisting the relative motion of solid surfaces, fluid layers and material elements sliding against each other. Friction force is proportional to the apparent weight :

$$
f_{F}=\mu f_{w}
$$

where $\mu$ is the coefficient of friction.

3) Magnetic force and torque: The magnetic force and torque that are exerted on an object with uniform magnetization $\vec{M}$ in a magnetic field with flux density $\vec{B}$ are defined as [14] :

$$
\begin{gathered}
\overrightarrow{f_{m}}=V_{m}(\vec{M} \cdot \nabla) \vec{B} \\
\overrightarrow{\tau_{m}}=V_{m} \vec{M} \times \vec{B}
\end{gathered}
$$

where $V_{m}$ is volume of the magnetic part of SHN. An applied magnetic field $\vec{B}$ at the location of the magnetic SHN transduces into torque $\tau_{m}$ and spatial magnetic gradient $\nabla \vec{B}$ transduces into applied force $\overrightarrow{f_{m}}$. 


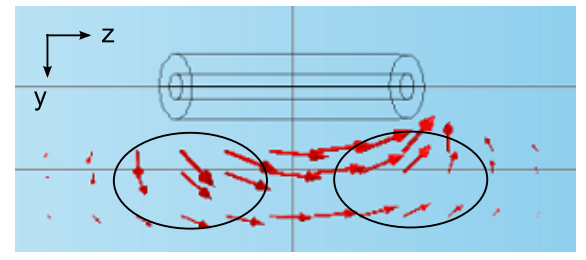

(a)

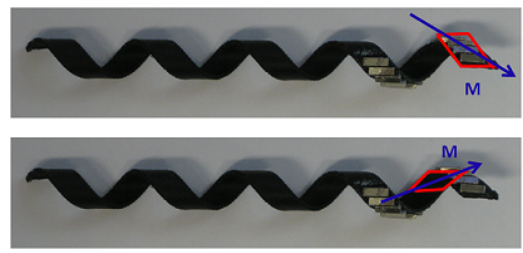

(d)

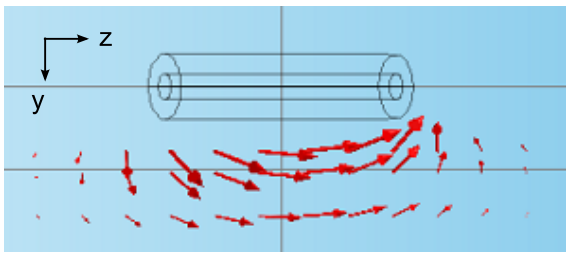

(b)

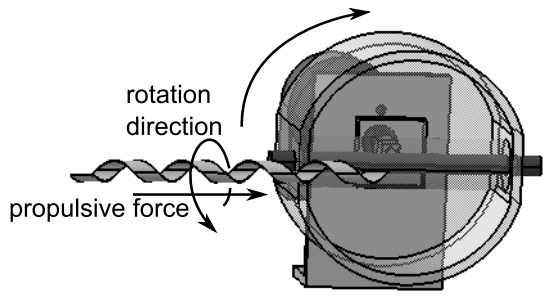

(e)

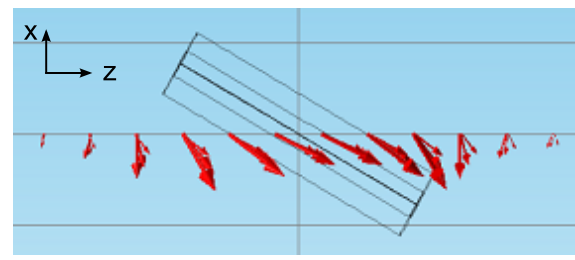

(c)

Fig. 4: (a) Magnetic field in $y O z$ plane generated by the magnet at the position $0^{\circ}$. (b) Magnetic field in $y O z$ plane generated by the magnet at the position $30^{\circ}$.(c) Magnetic field in $x O z$ plane generated by the magnet at the position $30^{\circ}$. (d) Two ways to consider the local magnetization of the helical tail : when SHN1 is located in the left-hand side (top) or the right-hand side (bottom) of the magnet. (e) The SHN1 in the left-hand side of magnet rotates in counter clockwise direction seen by its head. (f) The SHN1 in the right-hand side of magnet rotates in clockwise direction seen by its head.

\section{CharaCterization OF SCALED-UP HELICAL NANOBELT}

\section{A. Propulsion characteristics of SHNs}

Step-out frequencies of helical robot in their rotating propulsions have been revealed in [5], [8]. Researchers found that the forward velocity grows linearly with the applied field until a step-out frequency. Beyond this step-out frequency, the available magnetic torque is no longer sufficient to keep the microrobot rotating in sync with the applied field, resulting in a smooth but drastic decrease in velocity.

For SHNs, we observed rather a cut-off frequency. The SHNs rotate in sync with the applied magnetic field until the cut-off frequency is reached. Beyond this cut-off frequency, SHNs suddenly do not rotate anymore, but oscillate.

The cut-off frequency strongly depends on the viscosity of the liquid in which SHNs swim, because SHNs have to overcome the rotational fluidic drag torque which depends on the viscosity of the liquid. Different cut-off frequencies of SHN1 in different viscous liquids are depicted by Fig 5a, and that of SHN4 is depicted by Fig $5 b$.

The apparent weight of SHN4 is bigger than that of SHN1. The friction is proportional to the apparent weight. Therefore, more torque is required to rotate SHN4 than SHN1 with the same rotation frequency, as can be seen on Fig. 5. The rotating magnet provides the same magnetic torque to SHN1 and SHN4. Thus, cutoff frequency of SHN4 is smaller than that of SHN1 in

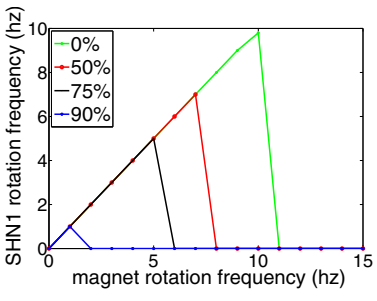

(a)

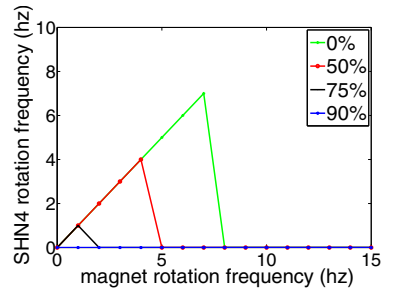

(b)
Fig. 5: Experimentally measured cut-off frequencies for (a) SHN1 and (b) SHN4.

the same viscous liquid for the same rotation frequency.

Two methods are viable for surpassing the cut-off frequency : to increase the magnetic torque by increasing the magnetic field magnitude and to reduce the helical belt weight. The method of increasing the magnetic field magnitude is the most suitable for microscale.

\section{B. Propulsion matrix identification}

The a, b, c parameters in (2) need be identified. The sign convention is defined as that the downward direction is positive for $f$ and $v$. The right-handed chirality rotation direction is defined as positive for $\omega$ and $\tau$.

The first experiment consists in dropping SHN1 freely in the $75 \%$ glycerol solution by placing the tube vertically. According to the parameters of SHN1 in Table I, its gravity force is measured as $0.02 \mathrm{~N}$ and the buoyancy force due to $75 \%$ glycerol solution is 
calculated as $0.01 \mathrm{~N}$ from the measured volume $(1 \mathrm{~mL})$. The nonfluidic applied force is the apparent weight calcuted as $0.01 \mathrm{~N}$. The measured dropping velocity is $102 \mathrm{~mm} / \mathrm{s}$ and the measured angular speed is $5.7 \mathrm{rad} / \mathrm{s}$.

If the SHN had a certain negative angular speed, its dropping velocity could be zero, because the propulsive force and buoyancy force would balance the gravity force. Unfortunately, below the cut-off frequency, this balance of forces is not reached.

The second experiment consists in dropping down SHN1 within an active rotating magnetic field. The external magnetic field gives a negative torque to SHN1. SHN1 drops with left-handed chirality rotation. The dropping velocities decrease. By adjusting the rotation frequency of the applied magnetic field to modify the angular speed of SHN1, the falling down velocity of the latter can be modified (see Table III). The velocities and angular speeds are noted $v_{i}$ and $\omega_{i}$ for rotating magnetic field of $i \mathrm{hz}$.

We assumed that the magnetic forces for different rotating magnetic fields are identical. Then, we calculate the propulsion matrix from the measures of dropping with $0 \mathrm{~Hz}, 1 \mathrm{~Hz}$ and $2 \mathrm{~Hz}$ external rotating magnetic field. From (2), the nonfluidic applied force $f$ is expressed as :

$$
f=a v_{1}+b \omega_{1}=a v_{2}+b \omega_{2}
$$

with $v_{1}=83 \mathrm{~mm} / \mathrm{s}, v_{2}=74 \mathrm{~mm} / \mathrm{s}, \omega_{1}=-6.3 \mathrm{rad} / \mathrm{s}$ and $\omega_{2}=-12.6 \mathrm{rad} / \mathrm{s}$. Equation (7) gives us a relationship between $\mathrm{a}$ and $\mathrm{b}$, that $0.009 a=-6.3 b$. The nonfluidic force for the first experiment is the apparent weight $0.01 \mathrm{~N}$, can be expressed as :

$$
f_{0}=a v_{0}+b \omega_{0}=-71.4 b+5.7 b
$$

with $v_{0}=102 \mathrm{~mm} / \mathrm{s}$ and $\omega_{0}=5.7 \mathrm{rad} / \mathrm{s}$. The coefficient $\mathrm{b}$ is calculated as $-1.5 \times 10^{-4}$ and the coefficient a is calculated as 0.11 .

The magnetic torque for the first experiment is zero, and can be expressed as :

$$
\tau_{0}=b v_{0}+c \omega_{0}
$$

with $v_{0}=102 \mathrm{~mm} / \mathrm{s}$ and $\omega_{0}=5.7 \mathrm{rad} / \mathrm{s}$. The coefficient c is calculated as $2.7 \times 10^{-6}$. Therefore, the propulsion matrix for SHN1 in $75 \%$ glycerol solution is estimated as :

$$
\left[\begin{array}{ll}
a & b \\
b & c
\end{array}\right]=\left[\begin{array}{cc}
0.11 & -1.5 \times 10^{-4} \\
-1.5 \times 10^{-4} & 2.7 \times 10^{-6}
\end{array}\right]
$$

We remark that all of the propulsion matrix coefficients are expressed in the international system of units.

This propulsion matrix of SHN1 in $75 \%$ glycerol solution estimated from the measures of dropping with $0 \mathrm{~Hz}, 1 \mathrm{~Hz}$ and $2 \mathrm{~Hz}$ external rotating magnetic field was verified by the measures of dropping with $3 \mathrm{~Hz}$ and $4 \mathrm{~Hz}$ external rotating magnetic field. The nonfluidic forces and torques can now be calculated and showed in Table III for dropping with different external

\begin{tabular}{|c|c|c|c|c|}
\hline Type & Viscosity & a & b & c \\
\hline \multirow{2}{*}{ SHN1 } & $50 \%$ & 0.06 & $-1.3 \times 10^{-4}$ & $1.8 \times 10^{-6}$ \\
& $75 \%$ & 0.11 & $-1.5 \times 10^{-4}$ & $2.7 \times 10^{-6}$ \\
& $90 \%$ & 0.73 & $-8.4 \times 10^{-4}$ & $1.1 \times 10^{-5}$ \\
\hline \multirow{2}{*}{ SHN4 } & $50 \%$ & 0.09 & $-1.2 \times 10^{-4}$ & $1.9 \times 10^{-6}$ \\
& $75 \%$ & 0.19 & $-2.9 \times 10^{-4}$ & $3.3 \times 10^{-6}$ \\
\hline
\end{tabular}

TABLE IV: Summary of $a, b$ and c parameters of propulsion matrices for the two types of SHNs in different viscous liquids. The fact that SHN1 and SHN4 drop fast in $50 \%$ glycerol solution can enlarge the inaccuracy of parameters estimation.

rotating magnetic field. According to the calculations, the nonfluidic forces are the gravity forces. Thus, the external rotating magnetic field is considered to give a pure magnetic torque, because there is no relative displacement between magnetic pitch and rotating magnet manipulator. We need thus two measurements to determine the $a, b, c$ parameters.

The same experiments, dropping freely and dropping with an external rotating magnetic field translating with the first pitch of SHN1 in 50\% and 90\% glycerol solutions, give us the propulsion matrices of SHN1 in 50\% and $90 \%$ glycerol.

The gravity force of SHN4 is measured as $0.05 \mathrm{~N}$ and the buoyancy force due to $75 \%$ glycerol solution is calculated as $0.015 \mathrm{~N}$ from the measured volume. The nonfluidic applied force is its apparent weight calcuted as $0.035 \mathrm{~N}$. The method to determine the propulsion matrices of SHN4 is the same. The propulsion matrix of SHN4 in 90\% glycerol solution cannot be estimated, because the cut-off frequency of SHN4 in 90\% glycerol solution is $0 \mathrm{~Hz}$. SHN4 drops very fast in $50 \%$ glycerol solution. This fact can enlarge the inaccuracy of parameters estimation. Table IV gathers the $a, b$ and $c$ parameters of propulsion matrices of SHN1 and SHN4 in different viscous liquids.

We observe that the absolute values of $a, b$, and $c$ parameters increase with viscosity. The a parameters of propulsion matrices of SHN4 are bigger than that of SHN1, and the c parameters are similar in propulsion matrices of SHN1 and of SHN4. These propulsion matrices will allow us to achieve a closed-loop control in the future.

\section{Force and torque analyses}

Forces and Torques are now simulated by the propulsion matrices estimated in section IV-B. Fig. 6a depicts the required forces and torques for SHN1 in 50\% glycerol solution in function of the rotation frequency for different desired translation velocities.

Less force is required for a higher rotation frequency with the same translation velocity, but more force should be applied on SHN1 for a higher translation velocity with the same rotation frequency. In ideal conditions, SHN1 rotating at $1.5 \mathrm{~Hz}$ can reach a translation 


\begin{tabular}{|c|c|c|c|c|c|c|c|c|c|c|c|c|c|}
\hline Type & \multicolumn{5}{|c|}{ SHN1 75\% } & \multicolumn{2}{|c|}{ SHN1 50\% } & \multicolumn{2}{|c|}{ SHN1 90\% } & \multicolumn{2}{|c|}{ SHN4 50\% } & \multicolumn{2}{|c|}{ SHN4 75\% } \\
\hline Rotation frequency (hz) & 0 & 1 & 2 & 3 & 4 & 0 & 3 & 0 & 1 & 0 & 3 & 0 & 1 \\
\hline Vertical velocity $(\mathrm{mm} / \mathrm{s})$ & 102 & 83 & 74 & 66 & 52 & 213 & 147 & 15.3 & 6.4 & 432 & 370 & 208 & 171 \\
\hline Angular speed (rad/s) & 5.7 & -6.3 & -12.6 & -18.8 & 14.8 & -14.4 & -25.1 & 1.2 & -6.3 & 27.3 & -19 & 18.4 & -6.3 \\
\hline Nonfluidic force $\left(10^{-2} \mathrm{~N}\right)$ & 1.02 & 1.02 & 1.04 & 1.06 & 1.02 & 1.09 & 1.07 & 1.00 & 1.00 & 3.56 & 3.56 & 3.44 & 3.45 \\
\hline Nonfluidic torque $\left(10^{-5}\right.$ N.m) & $\approx 0$ & -3.44 & -5.24 & -7.04 & -8.72 & $\approx 0$ & -4.50 & $\approx 0$ & -7.45 & $\approx 0$ & -8.05 & $\approx 0$ & -7.06 \\
\hline
\end{tabular}

TABLE III: Measurements of dropping velocities and angular speeds of SHN1 and SHN4 in different glycerol solutions in function of the rotation frequency of the applied magnetic field, and the nonfluidic forces and torques calculated from estimated propulsion matrices.

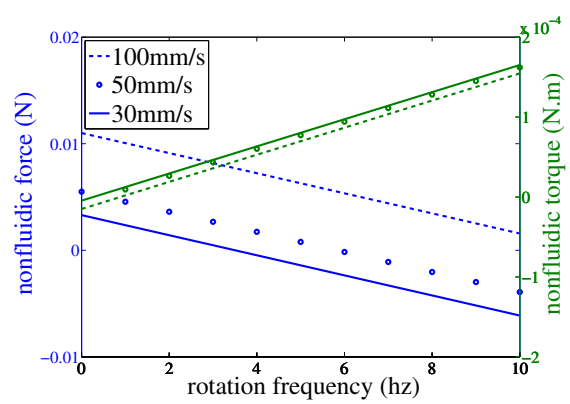

(a)

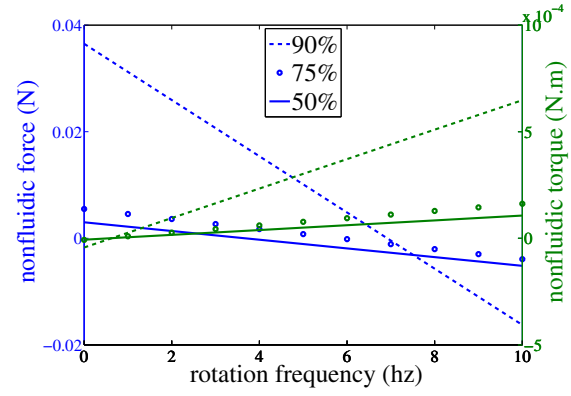

(b)

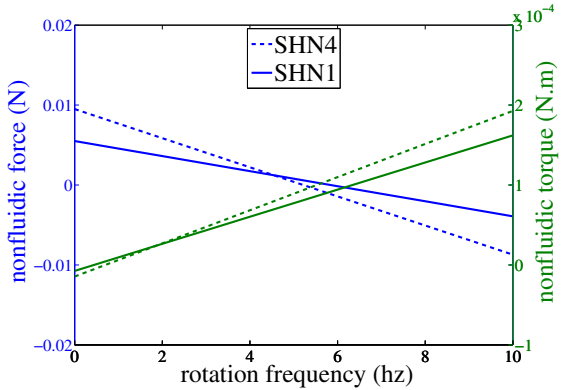

(c)

Fig. 6: (a) Required forces and torques for SHN1 in 50\% glycerol solution in function of SHN1's rotation frequency for different desired translation velocities. (b) Required forces and torques for translating SHN1 at $50 \mathrm{~mm} / \mathrm{s}$ in different viscous liquids. (c) Required forces and torques for SHN1 and SHN4 in 75\% glycerol solution in function of SHN1's rotation frequency for a desired translation velocity at $50 \mathrm{~mm} / \mathrm{s}$.

at $30 \mathrm{~mm} / \mathrm{s}$ without applied forces, because the pitch of SHN1 is $20 \mathrm{~mm}$. Yet in the experimental conditions, SHN1 had to rotate at around $2 \mathrm{~Hz}$ to keep a $30 \mathrm{~mm} / \mathrm{s}$ translation. This additional rotation frequency is to counterbalance the friction with the tube wall. SHN1 should rotate at $3 \mathrm{~Hz}-4 \mathrm{~Hz}$ instead of $2.5 \mathrm{~Hz}$ for a $50 \mathrm{~mm} / \mathrm{s}$ translation without applied forces, and $6 \mathrm{~Hz}$ - $7 \mathrm{~Hz}$ instead of $5 \mathrm{~Hz}$ for $100 \mathrm{~mm} / \mathrm{s}$. For $30 \mathrm{~mm} / \mathrm{s}$ and $50 \mathrm{~mm} / \mathrm{s}$ translation, the ranges of rotation frequency are confirmed by experiments. SHN1 can not reach the desired $100 \mathrm{~mm} / \mathrm{s}$ translation velocity in experiments below the cut-off frequency at $7 \mathrm{~Hz}$.

More torque is required for a higher rotation frequency. Theoretically, the required torque for the same rotation frequency does not depend on the translation velocity, as can be seen in Fig. 6a. Naturally, a helix translates screw-likely in a viscous liquid. Therefore a negative torque is required to keep SHN1 translating without rotation.

Fig. $6 \mathrm{~b}$ describes the required torques and forces for a translating SHN1 at $50 \mathrm{~mm} / \mathrm{s}$ in different viscous liquid. The figure shows that more torque and force are required for rotating SHN1 in a more viscous liquid, because more torque and force are needed to overcome the rotational fluidic drag torque and fluidic drag force.

The three torque lines cross at about $0.8 \mathrm{~Hz}$ and a little higher than $0 \mathrm{Nm}$. It means that if SHN1 is pulled with a pure force without torque and its translation velocity is $50 \mathrm{~mm} / \mathrm{s}$, it rotates at about $0.8 \mathrm{~Hz}$. The three

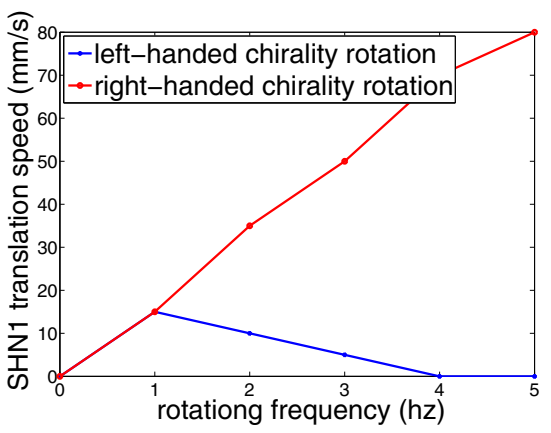

Fig. 7: Maximal translation speed for SHN1 in 75\% glycerol solution both for right-handed and left-handed chirality.

force lines cross $0 \mathrm{~N}$ with different rotation frequencies. In different viscous liquids, the resistances are not the same. Different additional rotation frequencies are required to counterbalance the different resistances.

Fig. $6 \mathrm{c}$ describes the required forces and torques for SHN1 and SHN4 in 75\% glycerol solution in function of the rotation frequency for a $50 \mathrm{~mm} / \mathrm{s}$ translation velocity. More force is required for rotating SHN4 than SHN1. The required torque for SHN4 and for SHN1 is closed, and the required torque for SHN4 is a little higher than that for SHN1. The influence of viscosity on torque is more important than the influence of weight. 


\section{Translation velocity of SHNs}

SHNs can swim forward following the rotating magnet manipulator in translation, depending on the balance of propulsive, magnetic, and friction force. The propulsive force is positive for right-handed chirality rotation, and negative for left-handed chirality rotation. Fig. 7 describes the maximal translation velocity of SHN1 in 75\% glycerol solution for different rotation frequencies of the magnetic field. The maximal translation velocity of SHN grows approximatively linearly with the rotation frequency of magnetic field until the cut-off frequency for right-handed chirality rotation, because the propulsive force of SHN1 grows.

SHN1 can also swim in left-handed chirality way, because the magnetic force overcomes the propulsive force and friction. Once the propulsive force increases and the sum of propulsive force and friction becomes bigger than the magnetic force, the SHN1 stops swimming in left-handed chirality way. Thus, the maximal translation velocity of left-handed chirality decreases with the rotation frequency.

\section{CONCLUSION}

In this paper, we proposed a scaled up experimental system for preliminary analysis of helical nanobelt swimming abilities. This scaled up system includes a SHN, permanent rotating magnet and linear translation to study the control parameters. We identified the propulsion matrices of SHN1 and SHN4 in different viscous liquids. The absolute values of $a, b, c$ parameters from the propulsion matrices increase with viscosity. The a parameters of propulsion matrices of SHN4 are bigger than that of SHN1, and the c parameters are similar in propulsion matrices of SHN1 and of SHN4. Less force is required for a higher rotation frequency to keep a SHN translating at a defined velocity. More force is required for a higher translation velocity. More force is required as well for a SHN in a more viscous liquid with a defined rotation frequency and translation velocity. Both more force and more torque are required for SHN4 than for SHN1 in the same condition with the same rotation frequency and translation velocity. Required torques are independent from the translation velocity. Required torques depend linearly on the rotation frequency and depend strongly on the viscosity of liquid. More torque is required for a higher rotation frequency in a more viscous liquid. The influence of viscosity of the liquid for torques is more important than the influence of the weight. These propulsion matrices allow us to achieve a closed-loop control in the future.

\section{ACKNOWLEDGMENT}

We acknowledge funding from Émergence-UPMC2011 research program

\section{REFERENCES}

[1] B.J. Nelson, I.K. Kaliakatsos, and J.J. Abbott. Microrobots for minimally invasive medicine. Annual Review of Biomedical Engineering, 12(1) :55-85, 2010.

[2] E.M. Purcell. Life at low reynolds number. American Journal of Physics, 45(1) :3-11, 1977.

[3] J.J. Abbott, M. Cosentino Lagomarsino, L. Zhang, L. Dong, and B.J. Nelson. How should microrobots swim? The International Journal of Robotics Research, 28(11-12) :1434-1447, 2009.

[4] T. Honda, K.I. Arai, and K. Ishiyama. Micro swimming mechanisms propelled by external magnetic fields. Magnetics, IEEE Transactions on, 32(5) :5085 -5087, sep 1996.

[5] K. Ishiyama, K.I. Arai, M. Sendoh, and A. Yamazaki. Spiraltype micro-machine for medical applications. In Micromechatronics and Human Science, 2000. MHS 2000. Proceedings of 2000 International Symposium on, pages 65-69, 2000.

[6] G.M. Patel, G.C. Patel, R.B. Patel, J.K. Patel, and M. Patel. Nanorobot : a versatile tool in nanomedicine. Journal of Drug Targeting, 14(2) :63-67, 2006.

[7] L. Zhang, J.J. Abbott, L. Dong, B.E. Kratochvil, D. Bell, and B.J. Nelson. Artificial bacterial flagella : Fabrication and magnetic control. Appl. Phys. Lett., 94(6), 2009.

[8] L. Zhang, J.J. Abbott, L. Dong, K.E. Peyer, B.E. Kratochvil, H. Zhang, C. Bergeles, and B. J. Nelson. Characterizing the swimming properties of artificial bacterial flagella. Nano Letters, 9 :3663-3667, October 2009.

[9] L. Zhang, K.E. Peyer, and B.J. Nelson. Artificial bacterial flagella for micromanipulation. Lab Chip, 10 :2203-2215, 2010.

[10] A. Ghosh and P. Fischer. Controlled propulsion of artificial magnetic nanostructured propellers. Nano Letters, 9(6) :2243$2245,2009$.

[11] G. Hwang, S. Haliyo, and S. Régnier. Remotely powered propulsion of helical nanobelts. In Robotics Science and Systems, 2010.

[12] G. Hwang, R. Braive, L. Couraud, A. Cavanna, O. Abdelkarim, I. Robert-Philip, A. Beveratos, I. Sagnes, S. Haliyo, and S. Régnier. Electro-osmotic propulsion of helical nanobelt swimmers. The International Journal of Robotics Research, 30(7) :806-819, 2011.

[13] T.W.R. Fountain, P.V. Kailat, and J.J. Abbott. Wireless control of magnetic helical microrobots using a rotating-permanentmagnet manipulator. In Robotics and Automation (ICRA), 2010 IEEE International Conference, pages 576 -581, may 2010.

[14] K.B. Yesin, K. Vollmers, and B.J. Nelson. Modeling and control of untethered biomicrorobots in a fluidic environment using electromagnetic fields. International Journal of Robotics Research, 25(5-6) :527-536, 2006. 